\title{
EDITORIAL
}

\section{Biomarkers: the next generation}

\author{
The value of biomarkers - characteristics that are evaluated as indicators of normal or \\ pathogenic biological processes, or responses to an intervention - is widely appreciated, \\ but the number of qualified biomarkers is small. However, recent initiatives have \\ highlighted promising strategies for developing the next generation of biomarkers.
}

In 1987, lovastatin — the first member of the now highly successful statin class of cardiovascular drugs - was approved by the US FDA, based on the demonstration that it could lower plasma levels of low-density lipoprotein (LDL) cholesterol ${ }^{1}$. The approval was a pivotal point in decades of research on the complicated and sometimes controversial relationship between plasma cholesterol levels and the risk of death from cardiovascular disease, as it provided a far more effective approach to reducing LDL cholesterol levels than had previously been available ${ }^{1}$. In the following two decades, multiple long-term clinical trials involving tens of thousands of patients showed that lowering levels of LDL cholesterol with drugs in the statin class reduced the risk of heart attacks, stroke and death.

The statin story provides a clear illustration of the value of effective biomarkers in drug development and healthcare decision-making; the massive studies conclusively demonstrating the benefit of statins on cardiovascular outcomes would have been much less viable without the confidence provided by their readily observable effects on LDL cholesterol levels. Indeed, the utility of LDL cholesterol as a biomarker of cardiovascular risk is reflected in the fact that it is qualified by the FDA as a surrogate end point for use in clinical trials. However, although nearly 25 years have passed since the approval of lovastatin, the number of other biomarkers that are accepted for use in regulatory decision-making is still small, despite substantial advances in the understanding of disease biology.

With the aim of bringing consistency and transparency to efforts to improve the translation of research knowledge into effective biomarkers, the US Institute of Medicine issued a report ${ }^{2}$ in May that recommends a framework for the evaluation of biomarkers, focusing on chronic diseases such as cardiovascular disease. As well as proposing a three-step process for biomarker evaluation - analytical validation of the performance of the biomarker assay; qualification of the association between the biomarker and disease states; and contextual analysis of the specific use proposed - the report also recommends that the FDA should convene expert panels to evaluate biomarkers with a regulatory impact ${ }^{2}$. Notably, in case studies of several well-known biomarkers included to illustrate the use of the framework, only LDL cholesterol was considered to have data that sufficiently supported its use as a surrogate end point ${ }^{2}$, which highlights the challenges in providing the data needed for new biomarkers to be qualified for such applications.

In efforts to address such challenges, collaborative initiatives are now taking centre stage. For example, the results of the first set of studies by the Predictive Safety Testing Consortium (PSTC) - which involves multiple drug companies, non-profit organizations, the FDA and the European Medicines Agency (EMA) - are presented in the May issue of Nature Biotechnology (http://www.nature. com/nbt/focus/pstc/index.html). These studies describe the evaluation of seven urinary proteins as biomarkers for drug-induced kidney toxicity, which are now formally considered by the FDA and the EMA as qualified for use in regulatory decision-making in a preclinical setting. As well as providing much-needed tools for filtering out drug candidates likely to cause kidney toxicity at an early stage, the regulatory process established in the qualification of these biomarkers will be valuable in the development of biomarkers of organ injury in other contexts.

It is also noteworthy that the process for the PSTC biomarkers involved submission of data to both the FDA and the EMA through the voluntary exploratory data submission programme, which was established by the FDA in 2004 with the aim of enhancing understanding of the regulatory significance of genomic data by both regulators and sponsors. To illustrate lessons learned in the first 5 years of this programme, the Perspective on page 435 of this issue presents a selection of case studies, also including newer platforms for generating biomarker data, such as microarray gene expression analysis. Given the additional scientific and technological complexity associated with such platforms compared with established approaches such as the levels of single proteins, it seems that collaborations such as that exemplified by the PSTC will have a key role in the qualification of the next generation of biomarkers for drug safety and efficacy.

\footnotetext{
Tobert, J. A. Lovastatin and beyond: the history of the HMG-CoA reductase inhibitors. Nature Rev. Drug Discov. 2, 517-526 (2003). 2. Institute of Medicine. Evaluation of biomarkers and surrogate endpoints in chronic disease. IOM website [online], http://www.iom.edu/Reports/2010Evaluation-of-Biomarkers-andSurrogate-Endpoints-in-Chronic-Disease.aspx (2010).
} 